\title{
Serotype variation in Streptococcus anginosus, S. constellatus and $S$. intermedius
}

\author{
M. INOUE, HIROKO EIFUKU-KOREEDA, K. KITADA, NOBUMI TAKAMATSU-MATSUSHITA, \\ YASUKO OKADA and E. OSANO*
}

Department of Preventive Dentistry, Kagoshima University Dental School, 8-35-1 Sakuragaoka, Kagoshima 890 and *Department of Oral Microbiology, Aichi-Gakuin University School of Dentistry, Nagoya 464, Japan

\begin{abstract}
Various serotypes reported in three serotyping systems for the 'Streptococcus milleri' group, i.e., Ottens I-IV, Osano I-IV and serotypes $a-k$, were compared with each other in a capillary precipitation test and by the double immunodiffusion test. Only two of the 19 serotypes, $f$ and Ottens-III, were identical, and the other 17 serotypes were independent. Thus, 18 serotypes and another three candidates ( $a$-, $b$ - and $g$-crossreactive) were found in the ' $S$. milleri' group. Of the 248 strains tested, 197 were serotypable, and 23 strains carried two type antigens. Generally, the Ottens I, II, III (serotype $f$, IV and Osano IV antigens, often together with the Lancefield group F antigen, were found in $S$. anginosus and $S$. constellatus. In addition, the serotype $a, c, d$, $e$ and $k$ antigens with the group $A, C$ or $G$ antigen were distributed in $S$. anginosus, the serotype $b$ antigen in $S$. constellatus and the $g, h, i, j$, Osano I, II and III antigens in $S$. intermedius. Twenty-five of the untypable strains were Lancefield groupable (mostly F).
\end{abstract}

\section{Introduction}

The 'Streptococcus milleri' group comprises a part of the normal flora in the human mouth and is often associated with serious pyogenic infections in various body sites [1-3]. These viridans streptococci consist of three distinct species, Streptococcus anginosus, $S$. constellatus and S. intermedius [4]. 'S. milleri' strains are known to show a wide serological variation, on the basis of their cell surface carbohydrate antigens, distinct from Lancefield group antigens. Ottens and colleagues first demonstrated the occurrence of five carbohydrate antigens $\mathrm{I}-\mathrm{V}$ (designated Ottens $\mathrm{I}-\mathrm{V}$ ) shared among 'indifferent' streptococci, including those belonging to Lancefield groups and also non-groupable strains [5-7]. More recently, Osano et al. reported that $S$. intermedius has five carbohydrate antigens $\mathrm{I}-\mathrm{V}$ (Osano I-V) [8]. Other studies also have demonstrated the presence of 11 serotypes $(a-k)$ among ' $S$. milleri' isolates from the mouth and various systemic infections [9-11], and the distribution of each serotype antigen is generally restricted to strains of a species in the ' $S$. milleri' group [12, 13].

Some of the carbohydrate serotype antigens found in ' $S$. milleri' strains have been characterised immuno-

Received 10 June 1997; accepted 22 Sept. 1997. Corresponding author: Dr M. Inoue. chemically [14-22]. However, relationships between these serotypes described independently in the serotyping systems remain generally undetermined. The present study examined the distribution of the 19 serotype antigens, $a-k$, Ottens I-IV and Osano I-IV, in combination with Lancefield group antigens, among 248 strains of the species within the ' $S$. milleri' group, and determined the immunological specificity of these serotypes in a capillary precipitation test and by immunodiffusion in an agar gel.

\section{Materials and methods}

'S. milleri' group strains

A total of 248 strains of ' $S$. milleri' group was examined. These included 18 ATCC or NCTC reference strains (including three substrains of NCTC 10708 ) and 230 clinical isolates from our laboratories $[8-10,23]$. These included the type strains of serotypes $a-k[10,11]$, Osano I-IV [8] and Ottens I-IV $[6,21]$ (Table 1), together with $S$. anginosus ATCC $33397^{\mathrm{T}}, S$. constellatus ATCC $27823^{\mathrm{T}}$ and $S$. intermedius ATCC $27335^{\mathrm{T}}$. These strains had been or were speciated on the basis of glycosidase production as described previously [12] and some had been verified genetically [13]. The 19 serotypes have been demonstrated previously to be distinct from Lancefield groups A-G $[6,8,11]$. 
Table 1. The type strains and typing antisera of the serotypes in the ' $S$. milleri' group

\begin{tabular}{|c|c|c|c|}
\hline Serotypes & $\begin{array}{l}\text { Type strains } \\
\text { (immunised with cells of) }\end{array}$ & \multicolumn{2}{|c|}{$\begin{array}{l}\text { Typing antisera } \\
\text { (absorbed with cells of) }\end{array}$} \\
\hline \multicolumn{4}{|c|}{ Serotype $a-k[10,11]$} \\
\hline$a$ & FW73 & - & \\
\hline$b$ & NCTC 10708 & $\mathrm{~K} 214-2 \mathrm{~K}(k)$ & \\
\hline$c$ & K51Y & $\mathrm{K} 217 \mathrm{~K}(d)$ & \\
\hline$d$ & $\mathrm{~K} 215-1 \mathrm{~K}$ & - & \\
\hline$e$ & $\mathrm{~K} 226 \mathrm{~K}$ & - & \\
\hline$f$ & ATCC 9895 & - & \\
\hline$g$ & $\mathrm{~K} 1 \mathrm{~K}$ & $\mathrm{~K} 201 \mathrm{~K}(j)$ & (431: Osano III)* \\
\hline$h$ & $\mathrm{~K} 18 \mathrm{~K}$ & - & \\
\hline$i$ & $\mathrm{~K} 39 \mathrm{~K}$ & $\mathrm{~K} 16-1 \mathrm{~K}(h)$ & \\
\hline$j$ & $\mathrm{~K} 201 \mathrm{~K}$ & - & (431: Osano III $)^{*}$ \\
\hline$k$ & $\mathrm{~K} 214-2 \mathrm{~K}$ & NCTC $10708(b)$ & \\
\hline \multicolumn{4}{|c|}{ Osano I-IV [8] } \\
\hline I & ATCC 27335 & - & \\
\hline II & 414 & - & \\
\hline II & 431 & - & \\
\hline IV & 510 & - & \\
\hline \multicolumn{4}{|c|}{ Ottens I-IV $[6,21]$} \\
\hline I & NCTC 11062 & - & \\
\hline II & NCTC 11063 & - & \\
\hline III & NCTC 11064 & - & \\
\hline IV & NCTC 11065 & - & \\
\hline
\end{tabular}

-, not absorbed.

* In some experiments absorbed with 431 (Osano III) cells

\section{Immunological methods}

Cells of the ' $S$. milleri' strains were grown anaerobically at $37^{\circ} \mathrm{C}$ for $18 \mathrm{~h}$ in Brain Heart Infusion Broth (BBL Microbiology Systems, Cockeysville, MD, USA). Cell surface carbohydrate antigens were extracted from glucose-grown cells by the method of Rantz and Randall [24].

The typing antisera for the serotypes $a-k[10,11]$ and the Osano I-IV [8] have been prepared previously. The Ottens I-IV typing antisera were raised in rabbits with glucose-grown cells of strains NCTC 11062, NCTC 11063, NCTC 11064 and NCTC 11065 by the procedures reported previously [11]. When required, a typing serum was absorbed with glucose-grown cells of an appropriate strain as described previously [10]. The Lancefield group A-G typing antisera and the group $\mathrm{F}$ antigen preparation were purchased from Difco Laboratories (Detroit, MI, USA).

Reactivity of antigen extracts with the typing antisera was examined in a capillary precipitin test [25]. The double immunodiffusion analysis was performed in a Noble Agar (Difco) $1.2 \%$ gel in $0.01 \mathrm{M}$ phosphate buffer, $\mathrm{pH} 7.2$, as described previously [11].

\section{Results and discussion}

Of the 248 strains tested, 197 were reactive or crossreactive with at least one of the 19 typing antisera, and 32 and 10 strains of the serotypable isolates reacted with two and three antisera, respectively, mostly in combinations of serotype $f$ and Ottens III, serotype $g$ or $j$ and Osano III, and $g$ - and $j$-cross-reactive $(g, j-)$ and Osano III (Fig. 1).

The antigen extracts of all the serotype $f$ strains tested reacted with the anti-Ottens III serum and vice versa (Fig. 1). Incubation in homologous and heterologous combinations of the extracts from the serotype $f$ and Ottens III type strains with their typing antisera produced a completely fused precipitin line (Fig, 2), indicating that these two, differently designated antigens, are identical.

Only the extracts from the serotypes $g, j$ and $g, j$ cross-reactive strains were reactive with the Osano III typing antiserum (Fig. 1). However, the $g$ and $j$ typing antisera absorbed with the Osano III type strain 431 cells did not react with the extracts from the $g, j$ cross-reactive strains including 431 (Fig. 3).

The antigen extracts from three substrains of the serotype $b$ type strain NCTC 10708 were consistently reactive with the Osano II typing antiserum, but the extracts from the other serotype $b$ or Osano II strains were unreactive with the heterologous antiserum (Fig. 1). The precipitin lines formed by the extracts from NCTC 10708 with the two typing antisera crossed each other (data not shown).

These results indicate that the Osano III antigen is distinct from the serotype $g$ and $j$ antigens and the Osano II from the serotype $b$ antigen. The antigen extracts from the other serotypable strains were reactive or cross-reactive $(a-, b$ - or $g$-) with only a single antiserum respectively (Fig. 1). 


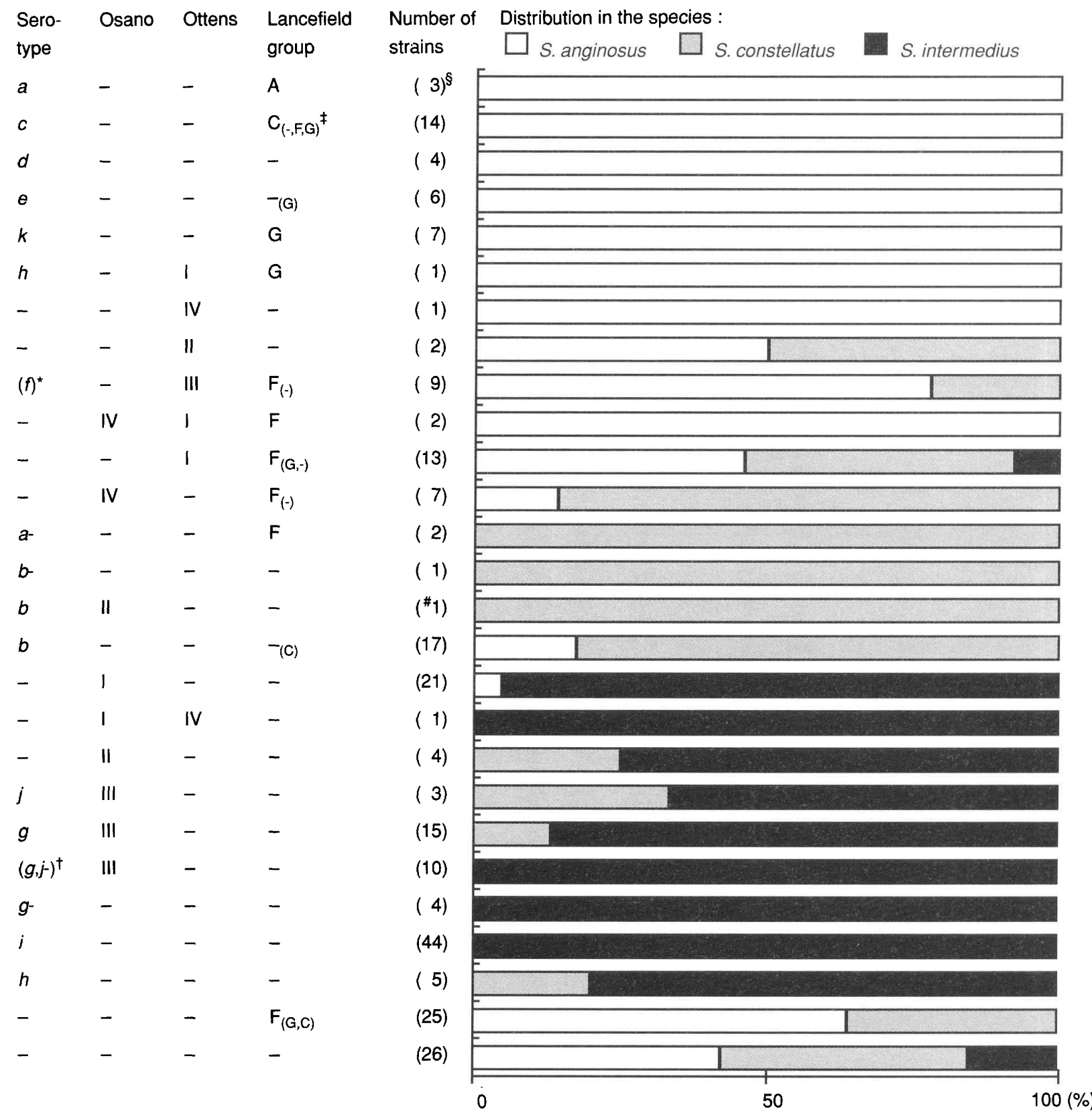

Fig. 1. Distribution of the serotype antigens in combination with Lancefield group antigens in the ' $S$. milleri' group: 85 $S$. anginosus strains, $57 \mathrm{~S}$. constellatus strains and $106 \mathrm{~S}$. intermedius strains were examined. -, untypable; $a-, b-, g-$, $g, j-: a-, b-, g-$, or $g, j$-cross-reactive; ( $)^{*}:$ identical to the Ottens III antigen (see Fig. 2 ); ( ) $\dagger:$ not detected by the $g$ and $j$ typing antisera absorbed with cells of the Osano III type strain 431 (see Fig. 3); ( ) or two strains; ( $)$ : number of strains tested; \#: tested with three substrains of $S$. constellatus NCTC 10708.

Taken together, 18 serotypes (one had two designations) and a further three candidates were demonstrated in the ' $S$. milleri' group. Twenty-three of the 197 serotypable strains had two different type antigens, mostly in combinations of serotype $g$ or $j$ and Osano III.

The Ottens I, II, III (or serotype f), IV and Osano IV antigens were found among $S$. anginosus and $S$. constellatus strains (Fig. 1). It was confirmed that the serotype $a, c, d, e$ and $k$ antigens are distributed among $S$. anginosus strains, the serotype $b$ antigen generally among $S$. constellatus strains, and the serotype $g, h, i, j$, Osano I, II and III antigens normally among $S$. intermedius strains $[8,12,13]$.

In addition, the Ottens antigens, particularly Ottens I and III (or serotype $f$ ), and also the Osano IV antigen were generally detectable with the group $\mathrm{F}$ antigen $[6,9,11]$ (Fig. 1). It was also confirmed that the group $\mathrm{F}$ antigen is found among $S$. anginosus and $S$. constellatus strains $[12,13]$ and that the group A, C and $\mathrm{G}$ antigens, which are present normally with the serotype $a, c$ and $k$ antigens, respectively, are restricted to distribution among $S$. anginosus strains $[9,11]$. 


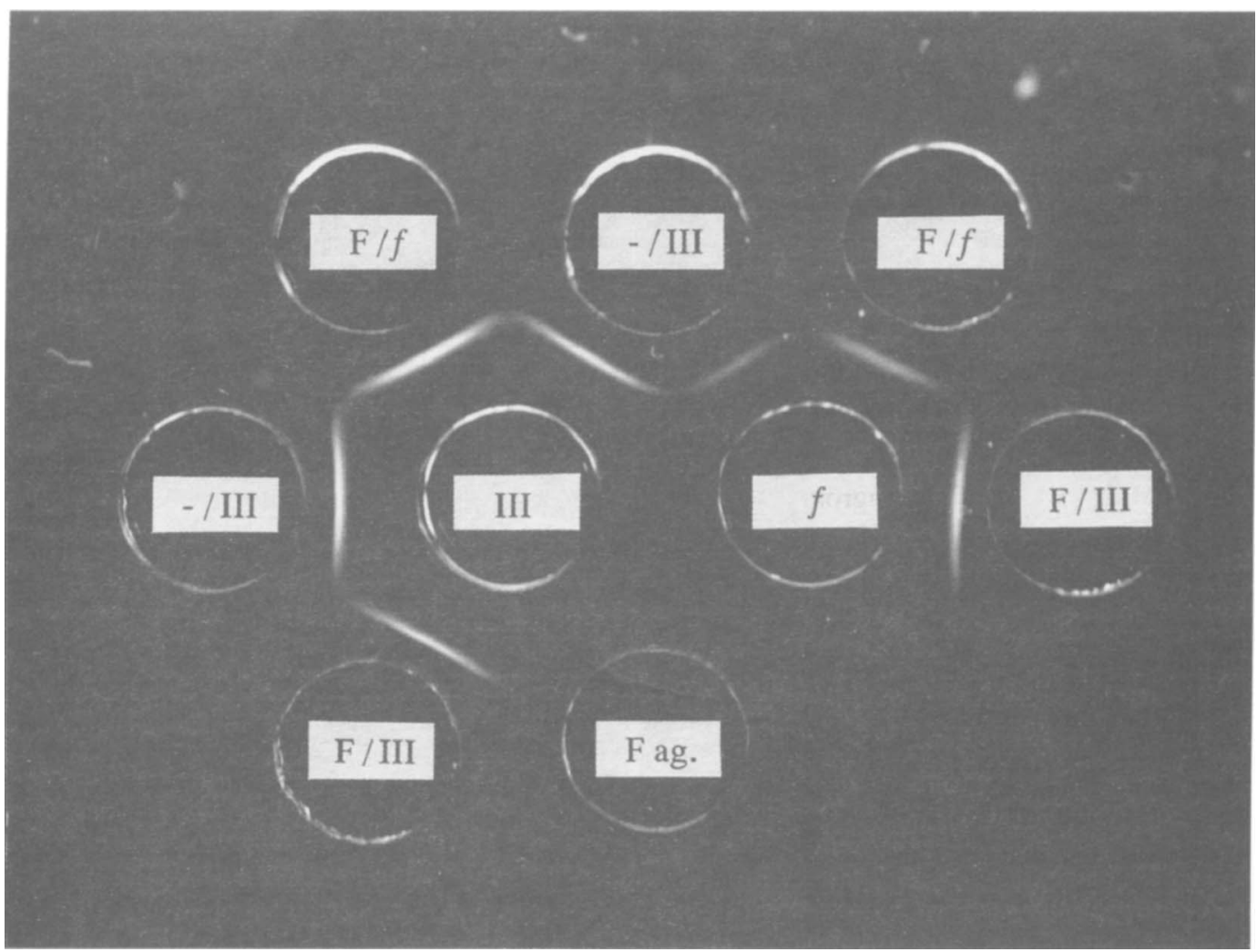

Fig. 2. Identity of the Ottens III and serotype $f$ antigens. Centre wells: III, the anti-Ottens III serum; $f$, the antiserotype $f$ serum. Peripheral wells: $-/$ III, the antigen extracts from NCTC 11064 ; F/III, NCTC 8037 or F/f, ATCC 9895 whole cells; F ag. Lancefield group $\mathrm{F}$ antigen.

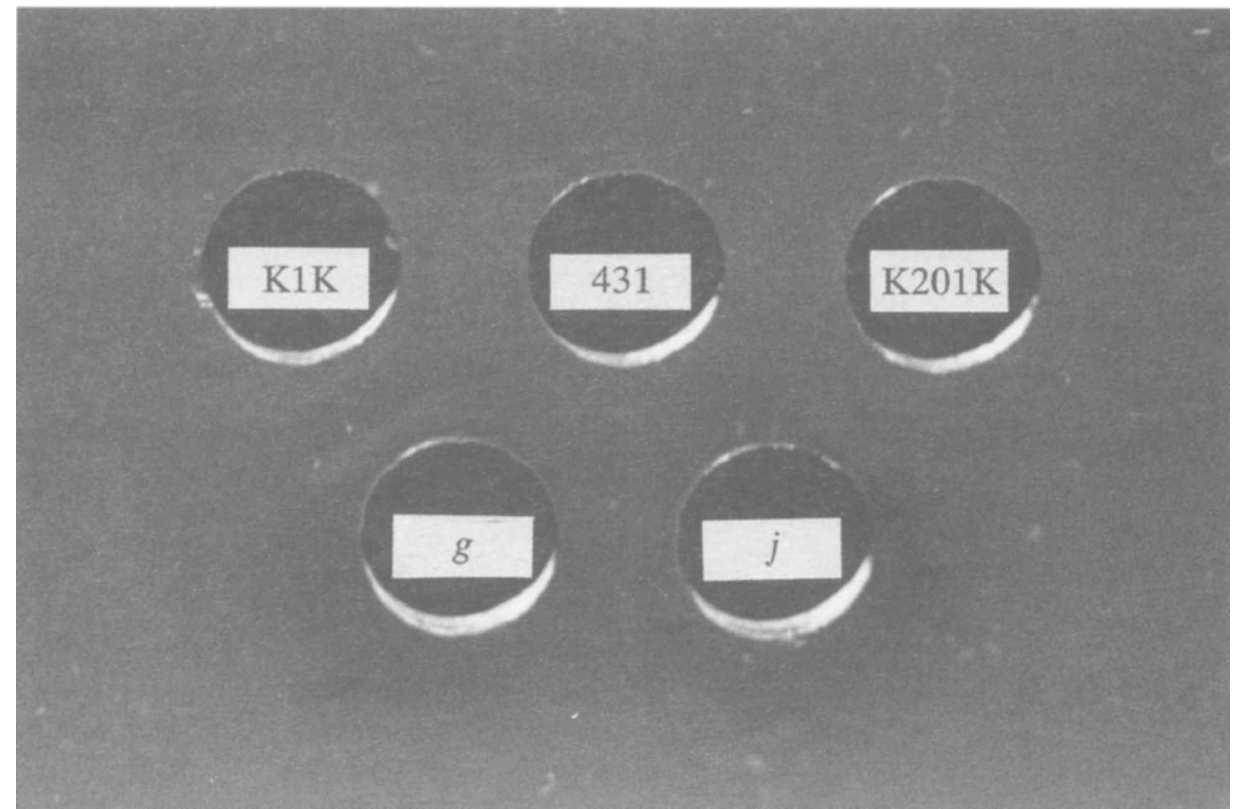

Fig. 3. Dissimilarity of the Osano III antigen from the serotype $g$ and $j$ antigens. Lower wells: the anti-serotype $g$ 'specific' serum $(g)$ and the anti-serotype $j$-'specific' serum $(j)$ which were absorbed with cells of the Osano III type strain 431. Upper wells: the antigen extracts from cells of the Osano III type strain 431 (431), the serotype $g$ type strain $\mathrm{K} 1 \mathrm{~K}(\mathrm{~K} 1 \mathrm{~K})$ and the serotype $j$ type strain K201K (K201K).

A few $S$. constellatus or $S$. intermedius strains were $a$-, $b$ - or $g$-cross-reactive and one-fifth of the ' $S$. milleri' strains tested remained untypable, although half the number were Lancefield groupable (mostly group F) (Fig. 1). The Ottens $\mathrm{V}$ and Osano $\mathrm{V}$ antigens reported in previous studies $[6,8]$ might be distributed among these strains and involved in any of the crossreactivities. Unfortunately, the possibilities cannot be verified because the type or reference strain or the typing antiserum preparation for these serotypes are 
not available. Some presently unknown antigens also exist among the ' $S$. milleri' group.

In conclusion, only two of the 19 serotypes described in the three serotyping systems were identical, and at least 18 serotypes apart from Lancefield groups are confirmed to exist in the ' $S$. milleri' group. Eight, three and seven of these serotypes were preferentially detectable in $S$. anginosus - serotypes $a, c, d, e, k$, Ottens I, III (or f), Osano IV - S. constellatus (b, Ottens I, Osano IV) and $S$. intermedius ( $g, h, i, j$, Osano I, II, III), respectively. One-tenth of the 248 strains tested remained untypable and Lancefield ungroupable.

The proposed serological scheme for the ' $S$. milleri' group is not fully established and the phenotypic characteristics determined by the antisera so far available are of little practical use for the correct speciation of ' $S$. milleri' strains. However, like some modern molecular techniques such as ribotyping, this simple and rapid serotyping method could be a classical but useful tool for typing individual strains of a given species within the ' $S$. milleri' group and provide additional data to clarify their route of transmission in clinical and epidemiological studies. It is important to examine whether some of these serotype antigens are involved in the pathogenicity of the ' $S$. milleri' group - studies are ongoing in our laboratory.

\section{References}

1. Gossling J. Occurrence and pathogenicity of the Streptococcus milleri group. Rev Infect Dis 1988; 10: 257-285.

2. Ruoff KL. Streptococcus anginosus ('Streptococcus milleri'): the unrecognized pathogen. Clin Microbiol Rev 1988; 1: $102-108$.

3. Whitworth JM. Lancefield group $\mathrm{F}$ and related streptococci. $J$ Med Microbiol 1990; 33: 135-151.

4. Whiley RA, Beighton D. Emended descriptions and recognition of Streptococcus constellatus, Streptococcus intermedius, and Streptococcus anginosus as distinct species. Int $J$ Syst Bacteriol 1991; 41: 1-5.

5. Jablon JM, Brust B, Saslaw MS. $\beta$-hemolytic streptococci with group A and type II carbohydrate antigens. J Bacteriol 1965 89: $529-534$

6. Ottens H, Winkler KC. Indifferent and haemolytic streptococci possessing group-antigen F. $J$ Gen Microbiol 1962; 28: $181-191$.

7. Willers JMN, Ottens H, Michel MF. Immunochemical relationship between Streptococcus MG, F III and Streptococcus salivarius. J Gen Microbiol 1964; 37: 425-431.
8. Osano E, Tanaka T, Ozeki M, Makita I, Moriyama T. Serogrouping of oral Streptococcus intermedius. Microbiol Immunol 1990; 34: 211-219.

9. Kitada $K$, Nagata $K$, Yakushiji T, Eifuku $H$, Inoue $M$. Serological and biological characteristics of 'Streptococcus milleri' isolates from systemic purulent infections. $J \mathrm{Med}$ Microbiol 1992; 36: 143-148.

10. Yakushiji T, Kitada K, Okita Y, Inoue M. Distribution of Streptococcus milleri in the oral cavities of Japanese children. Microb Ecol Health Dis 1990; 3: 171-179.

11. Yakushiji $\mathrm{T}$, Konagawa $\mathrm{R}$, Oda $\mathrm{M}$, Inoue $\mathrm{M}$. Serological variation in oral Streptococcus milleri. J Med Microbiol 1988; 27: $145-151$.

12. Taketoshi $M$, Kitada $K$, Yakushiji $T$, Inoue $M$. Enzymatic differentiation and biochemical and serological characteristics of the clinical isolates of Streptococcus anginosus, $S$. intermedius and S. constellatus. Microbios 1993; 76: 115-129.

13. Taketoshi $M$, Yakushiji $T$, Inoue $M$. Deoxyribonucleic acid relatedness and phenotypic characteristics of oral Streptococcus milleri strains. Microbios 1993; 73: 269-280.

14. Inoue $\mathrm{M}$, Yakushiji $\mathrm{T}$, Konagawa $\mathrm{R}$. Carbohydrate antigen of serotype g 'Streptococcus milleri': immunochemical characterization. Oral Microbiol Immunol 1991; 6: 295-298.

15. Kitada $\mathrm{K}$, Inoue $\mathrm{M}$. Immunochemical characterization of the carbohydrate antigens of serotype $\mathrm{k}$ and Lancefield group $\mathrm{G}$ 'Streptococcus milleri'. Oral Microbiol Immunol 1996; 11 22-28.

16. Kitada K, Yakushiji T, Inoue M. Immunochemical characterization of the carbohydrate antigens of serotype $\mathrm{c} /$ Lancefield group C 'Streptococcus milleri'. Oral Microbiol Immunol 1993; 8: $161-166$.

17. Konagawa R, Yakushiji $\mathrm{T}$, Inoue $\mathrm{M}$. Immunochemical characterization of type $\mathrm{i}$ carbohydrate antigen of 'Streptococcus milleri' (Streptococcus anginosus). Int J Med Microbiol 1990; 274: $40-49$.

18. Michel MF, van Vonno J, Krause RM. Studies on the chemical structure and the antigenic determinant of type II antigen of group F streptococci. J Immunol 1969; 102: 215-221.

19. Willers JMN, Michel MF, Benner R. Immunochemical studies of type IV and two group-like (Z) carbohydrate antigens of minute streptococci. Antonie van Leeuwenhoek 1973; 39: 609-617.

20. Willers JMN, Michel MF, Huis in'tVeld JHJ, Alderkamp GHJ. The type antigen III of group F streptococci. Separation of group and type antigens and partial characterization of type III antigen. Antonie van Leeuwenhoek 1973; 39: 369-382.

21. Willers JMN, Michel MF, Sysma MJ, Winkler KC. Chemical analysis and inhibition reactions of the group and type antigens of group F streptococci. J Gen Microbiol 1964; 36: 95-105.

22. Yakushiji T, Inoue $M$, Koga $T$. Purification and immunochemical studies of type b carbohydrate antigen of oral Streptococcus milleri. Infect Immun 1988; 56: 2264-2269.

23. Yakushiji T, Katsuki M, Yoshimitsu A, Mizuno J, Inoue $M$ Isolation and physiological characterization of Streptococcus milleri strains from human dental plaque. Microbios 1988; 55 : $161-171$

24. Rantz LA, Randall E. Use of autoclaved extracts of hemolytic streptococci for serological grouping. Stanford Med Bull 1955; 13: $290-291$

25. Inoue $\mathrm{M}$, Matsunoshita N, Yakushiji T. Restricted distribution of Streptococcus milleri carbohydrate type antigens amongst other viridans streptococci. $J$ Med Microbiol 1992; 37: $211-213$ 